\title{
Nonthrombogenic, Biodegradable Elastomeric Polyurethanes with Variable Sulfobetaine Content
}

\author{
Sang-Ho Ye, ${ }^{\dagger, \ddagger, \text { II }}$ Yi Hong, ${ }^{\dagger, \ddagger, \S, \text { IL }}$ Hirokazu Sakaguchi, ${ }^{\dagger, \perp}$ Venkat Shankarraman, ${ }^{\dagger}$ Samuel K. Luketich,"

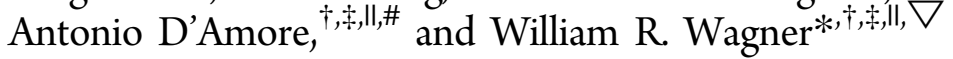

${ }^{\dagger}$ McGowan Institute for Regenerative Medicine, ${ }^{*}$ Department of Surgery, "Department of Bioengineering, and $\nabla_{\text {Department of }}$ Chemical Engineering, University of Pittsburgh, Pittsburgh, Pennsylvania 15219, United States

${ }^{\perp}$ Toray Industries Inc., Tokyo, Japan

"RiMED Foundation and DICGIM, University of Palermo, 90133 Palermo, Italy

\section{Supporting Information}

ABSTRACT: For applications where degradable polymers are likely to have extended blood contact, it is often important for these materials to exhibit high levels of thromboresistance. This can be achieved with surface modification approaches, but such modifications may be transient with degradation. Alternatively, polymer design can be altered such that the bulk polymer is thromboresistant and this is maintained with degradation. Toward this end a series of biodegradable, elastic polyurethanes (PESBUUs) containing different zwitterionic
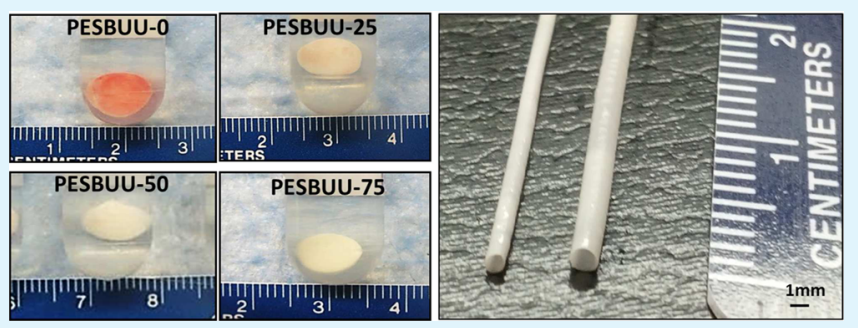
sulfobetaine $(\mathrm{SB})$ content were synthesized from a polycaprolactone-diol (PCL-diol):SB-diol mixture (100:0, 75:25, 50:50, 25:75 and 0:100) reacted with diisocyanatobutane and chain extended with putrescine. The chemical structure, tensile mechanical properties, thermal properties, hydrophilicity, biodegradability, fibrinogen adsorption and thrombogenicity of the resulting polymers was characterized. With increased SB content some weakening in tensile properties occurred in wet conditions and enzymatic degradation also decreased. However, at higher zwitterionic molar ratios (50\% and $75 \%)$ wet tensile strength exceeded $15 \mathrm{MPa}$ and breaking strain was $>500 \%$. Markedly reduced thrombotic deposition was observed both before and after substantial degradation for both of these PESBUUs and they could be processed by electrospinning into a vascular conduit format with appropriate compliance properties. The mechanical and degradation properties as well as the acute in vitro thrombogenicity assessment suggest that these tunable polyurethanes could provide options appropriate for use in blood contacting applications where a degradable, elastomeric component with enduring thromboresistance is desired.

KEYWORDS: biodegradable polyurethane, sulfobetaine, cardiovascular, thromboresistance, vascular graft, zwitterion

\section{INTRODUCTION}

A variety of approaches have been pursued to reduce the thrombogenicity of polymers intended for device applications with extended blood contact, including high hydrophobicity, ${ }^{1}$ high hydrophilicity, ${ }^{2}$ heparinization, ${ }^{3,4}$ zwitterion incorporation, ${ }^{5,6}$ and biomimetic design strategies. ${ }^{7,8}$ Incorporating zwitterionic groups, such as phosphorylcholine (PC), sulfobetaine $(\mathrm{SB})$ or carboxybetaine (CB) on blood contacting surfaces has been shown in a number of systems to be an effective and convenient approach to minimize protein adsorption and platelet deposition. ${ }^{6,9-11}$ SB-containing monomers are relatively cheap and easily obtained compared to the PC and CB, whereas surfaces modified with SB-containing molecules have exhibited antifouling and antithrombogenic properties similar to surfaces modified with other zwitterionic groups. ${ }^{12-14} \mathrm{SB}$ has often been used in strategies to reduce surface fouling on nondegradable biomaterials, ${ }^{15,16}$ whereas only recently has this zwitterion been considered for incorporation in blood contacting biodegradable polymers. ${ }^{17,18}$
Biodegradable polyurethane materials have been evaluated for potential use in several cardiovascular applications, including as scaffolds for tissue engineered vascular grafts and stent coatings. ${ }^{19-21}$ Surface modification, ${ }^{15,22}$ functional moiety incorporation, ${ }^{18,23}$ and controlled release of bioactive agents ${ }^{24}$ have been employed to improve polyurethane blood compatibility. For biodegradable polyurethane vascular scaffolds, PC surface modification and PC copolymer blending have exhibited good thromboresistance in vitro and in vivo. ${ }^{25,26}$ In another approach, designed to modify the polyurethane backbone throughout the bulk phase of the polymer, PC moieties were conjugated as pendant groups onto poly(ester urethane) ureas containing pendant $\mathrm{COOH}$ groups (PEUUPC). ${ }^{27,26}$ However, PEUU-PC polymers had a limited capacity for PC grafting due to limits on the extent of $\mathrm{COOH}$ group incorporation with the synthetic scheme employed.

Received: October 10, 2014

Accepted: November 21, 2014 
(A)<smiles>CCCCN(CCO)CCO</smiles><smiles></smiles>

(B)<smiles>CCCC[N+](CCO)(CCO)CCS(=O)(=O)[O-]</smiles><smiles>CCCCCNC(=O)NCCCCNC(=O)NCCCCNC(=O)NCCCCNC(=O)NCCCCNC(=O)OCCCCNC(=O)OCC[NH+](C)CCCS(=O)(=O)[O-]</smiles>

Figure 1. Schematic synthesis of (A) sulfobetaine-diol (SB-diol) and (B) biodegradable polyester sulfobetaine urethane ureas (PESBUUs).

In this study, biodegradable, elastic polyurethanes with $\mathrm{SB}$ incorporated into the polymer backbone were synthesized, where SB content in the polymer could be readily tuned by altering the molar ratio of diols employed in the synthesis. Specifically, SB with two hydroxyl end groups (SB-diol) was synthesized followed by blending of SB-diol and polycaprolactone-diol at different molar ratios prior to reaction with diisocyanatobutane followed by chain extension with putrescine. This approach is straightforward and does not require a subsequent step to graft on the biofunctional moieties. The chemical structure, mechanical properties, thermal properties, hydrophilicity, biodegradability, and thrombogenicity of the resulting polymers was characterized. Surface protein deposition was evaluated using fibrinogen as a model protein. Whole ovine blood was used to assess platelet deposition on the developed polyurethanes before and after degradation for maintenance of thromboresistance. One of the promising polymers was processed by electrospinning into a conduit that would be structurally appropriate for use as a vascular graft.

\section{EXPERIMENTAL SECTION}

Materials. $N$-Butyldiethanolamine and 1,3-propane sultone were purchased from Sigma-Aldrich and used without further purification. Polycaprolactone diol (number-average molecular weight 2000, PCLdiol, Sigma) was dried in vacuum oven at $60{ }^{\circ} \mathrm{C}$ prior to synthesis. Putrescine (Sigma) and diisocyanatobutane (BDI, Sigma) were purified by a vacuum distillation. Stannous octotate $\left(\mathrm{Sn}(\mathrm{Oct})_{2}\right)$ was dried using 4 Å molecular sieves (Sigma). Dimethyl sulfoxide (DMSO, anhydrous $\geq 99.9 \%$, Sigma), 1,1,1,3,3,3-hexafluoroisopropanol (HFIP,
Oakwood Inc.), and other chemicals used as received, except what mentioned above.

Synthesis of Sulfobetaine-diol. Sulfobetaine with two hydroxyl groups (SB-diol) was synthesized from 1,3-propane sultone (PS) and N-butyldiethanolamine (BDEA) (Figure 1A). Briefly, 0.3 M PS and BDEA were dissolved together in $200 \mathrm{~mL}$ of anhydrous methylene chloride in a round-bottom flask with a stir bar. After argon injection for $20 \mathrm{~min}$, the flask was sealed and the mixture stirred for $15 \mathrm{~h}$ at 40 ${ }^{\circ} \mathrm{C}$. The solvent was then removed from the reaction mixture using a rotary evaporator and a white precipitate was obtained. The product was washed several times with diethyl ether to remove unreacted species. The chemical structure was confirmed by proton nuclear magnetic resonance $\left({ }^{1} \mathrm{H}\right.$ NMR, BrukerBiospin Co., Billerica, MA). For SB-diol (in $\mathrm{D}_{2} \mathrm{O}$ ), the peaks were: $\delta$ (ppm) $0.85-0.95\left(\mathrm{CH}_{2} \mathrm{CH}_{3}\right)$, 1.30-1.40 ( $\left.\mathrm{CH}_{2} \mathrm{CH}_{2} \mathrm{CH}_{2}\right)$, 1.65-1.75 $\left(\mathrm{CH}_{2} \mathrm{CH}_{3}\right), \quad 2.10-2.20$ $\left(\mathrm{C}_{2} \mathrm{CH}_{2} \mathrm{SO}_{3}\right), 2.90-3.00\left(\mathrm{CH}_{2} \mathrm{SO}_{3}\right), 3.40-3.60\left(\left(\mathrm{CH}_{2}\right)_{4} \mathrm{~N}\right)$, 3.95-4.05 $\left(\mathrm{CH}_{2} \mathrm{OH}\right)_{2}$ (see the Supporting Information, Figure $\mathrm{S} 1$ ).

Synthesis and Processing of Polyurethane Ureas Containing Sulfobetaine (PESBUUs). PESBUUs were synthesized using a twostep solvent polymerization in a manner similar to that previously reported $^{28}$ with the synthesis scheme shown in Figure 1B. PCL-diol and SB-diol were blended at different molar ratios, respectively: 100:0, 75:25, 50:50, 25:75, and 0:100 (designated PESBUU-0, PESBUU-25, PESBUU-50, PESBUU-75, and PESBUU-100), and then were dissolved in DMSO at $70{ }^{\circ} \mathrm{C}$ in a three-necked flask with argon protection and agitation. BDI was added into the flask, following three droplets of catalyst $\mathrm{Sn}(\mathrm{Oct})_{2}$. The reaction was carried out for $3 \mathrm{~h}$ at $70{ }^{\circ} \mathrm{C}$, followed by cooling down the prepolymer solution to room temperature, and then adding a putrescine/DMSO solution dropwise into the flask. The reaction continued overnight, and then the polymer was precipitated in diethyl ether and dried in a vacuum oven at $60{ }^{\circ} \mathrm{C}$ for 3 days. The (PCL-diol+SB-diol):BDI:putrescine molar ratio was 


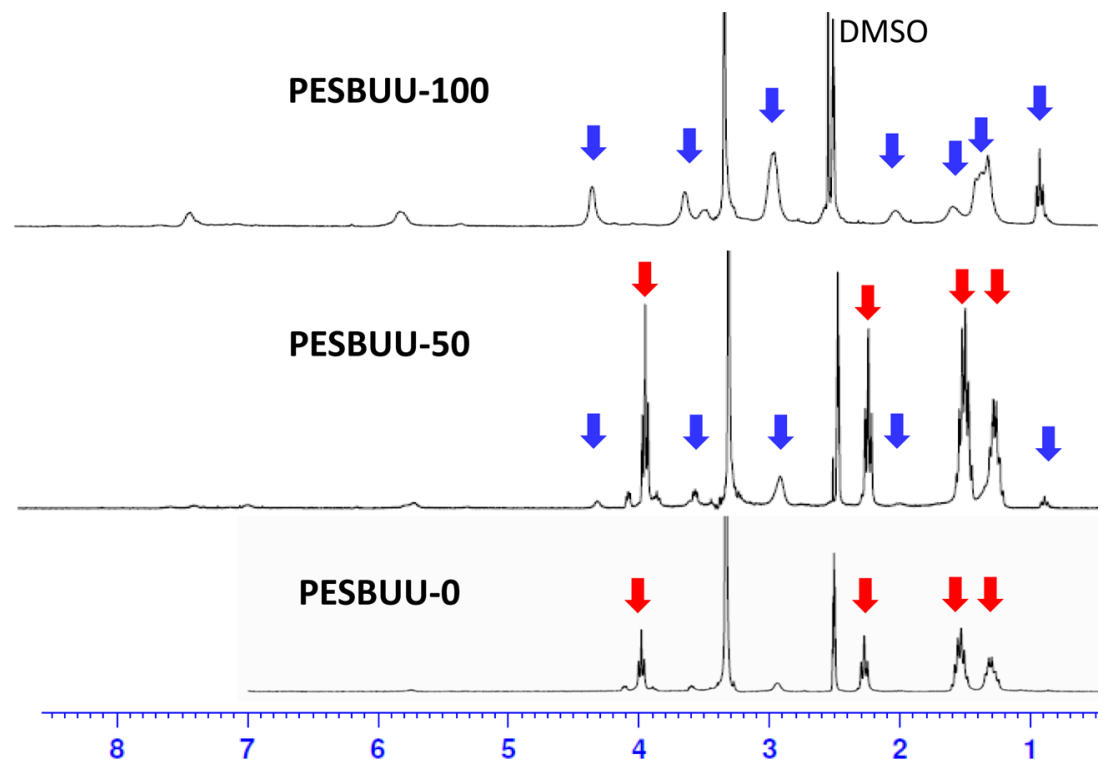

Figure 2. ${ }^{1} \mathrm{H}$ NMR spectra of PESBUU-0, PESBUU-50, and PESBUU-100. Peaks attributable to SB are designated with blue arrows and peaks attributable to PCL with red arrows.

set at 1:2:1. Polymer yields were above $80 \%$. Polymer films were fabricated by solvent casting using HFIP, followed by drying in a vacuum oven at $60{ }^{\circ} \mathrm{C}$ for 3 days.

Eletrospun fibrous sheets and conduits were fabricated from PESBUUs using methods previously described. ${ }^{25}$ Briefly, the PESBUU was dissolved in HFIP ( 8 wt \%) and the solution was fed at $1 \mathrm{~mL} / \mathrm{h}$ by syringe pump (Harvard Apparatus, U.S.) into a steel capillary (ID = $1.2 \mathrm{~mm}$ ) that was suspended $14 \mathrm{~cm}$ over a stainless steel mandrel (19 $\mathrm{mm}$ diameter for a sheet and 1.0 or $1.6 \mathrm{~mm}$ diameter for a conduit). The mandrel was rotated at $250 \mathrm{rpm}$ and rastered upon an $x-y$ stage (Velmex, U.S.) which was reciprocally translated in the direction of the mandrel axis at a speed of $6 \mathrm{~cm} / \mathrm{s}$ with an amplitude of $8 \mathrm{~cm}$. Two high-voltage generators (Gamma High Voltage Research, U.S.) were used to charge the polymer feeding capillary to $7 \mathrm{kV}$ and the mandrel to $-10 \mathrm{kV}$. The electrospun sheets and conduits were removed from the mandrels after electrospinning for $4 \mathrm{~h}$ for a sheet or $15 \mathrm{~min}$ for a conduit.

Polymer Characterization. ${ }^{1} \mathrm{H}$ NMR with DMSO- $d_{6}$ solvent was used to verify polymer chemical structure. Glass transition temperatures $\left(T_{\mathrm{g}}\right)$ and melting temperatures $\left(T_{\mathrm{m}}\right)$ were detected on a Shimazu differential scanning calorimetry (DSC, DSC-60, Shimazu) at a scanning range of -100 to $200{ }^{\circ} \mathrm{C}$ with a heating rate of $20^{\circ} \mathrm{C} / \mathrm{min}$ and a nitrogen flow. Intrinsic viscosity of the synthesized polymers was measured using an Ubbelohde viscometer at $22{ }^{\circ} \mathrm{C}$. ${ }^{28}$ Each polymer was dissolved in $20 \mathrm{~mL}$ of HFIP at a concentration of $0.6 \mathrm{~g} / \mathrm{dL}$ and then filtered using an $0.45 \mu \mathrm{m}$ polytetrafluoroethylene filter. The efflux time of each polymer sample and HFIP was measured to calculate the intrinsic viscosity as $\ln \left(t_{\mathrm{p}} / t_{\mathrm{s}}\right) / C_{\mathrm{p}}$, where $t_{\mathrm{p}}$ represents the polymer solution efflux time, $t_{\mathrm{s}}$ represents the HFIP efflux time, and $C_{\mathrm{p}}$ is the polymer concentration. Water absorption of polymer films was measured by immersing a weighted film $\left(W_{0}\right)$ in Dulbecco's phosphate buffered saline (DPBS, without calcium or magnesium) at $37{ }^{\circ} \mathrm{C}$ for $24 \mathrm{~h}$ and then weighting the wetted sample $\left(W_{1}\right)$. The water adsorption was calculated as $\left(W_{1}-W_{0}\right) / W_{0} \times 100 \%$.

Mechanical Testing. Mechanical properties of cast films were measured using an MTS Tytron 250 MicroForce Testing Workstation at room temperature with a crosshead speed of $25 \mathrm{~mm} / \mathrm{min}$. Five dumbbell-shaped strips $(2 \times 18 \mathrm{~mm})$ were cut from PESBUU-0, PESBUU-25, PESBUU-50, PESBUU-75 and PESBUU-100 polymer films and tested. The mechanical properties of polymers in wet conditions were measured after the dumbbell-shaped strips of PESBUUs films were immersed in DPBS at $37{ }^{\circ} \mathrm{C}$ for $24 \mathrm{~h}$. Cyclic tensile testing ${ }^{29}$ was conducted using a uniaxial cyclic tensile testing system (model PCI-MIO-16XE-10, National Instruments) to evaluate the elastic properties of the PESBUU polymers by stretching to maximum strains of $30 \%$ or $400 \%$ and retracing back to the initial length for 10 cycles at a rate of $150 \mathrm{~mm} / \mathrm{min}$.

The mechanical properties of electrospun fibrous sheets were also measured with a crosshead speed of $10 \mathrm{~mm} / \mathrm{min}$ on dumbbell-shaped strips of PESBUUs under wet conditions. For compliance testing of electrospun conduits, $1 \mathrm{~mm}$ diameter vessel segments, $18 \mathrm{~mm}$ in length were obtained from each PESBUU conduit with 3 samples/ group tested. The test circuit (see the Supporting Information, Figure S2A) was assembled using polyvinyl chloride tubing, connectors and stopcocks (Value Plastics, Inc.). Surgical suture (Henry Schein) was used to secure each segment to a Precision Guide needle (Becton Dickenson) $\sim 2 \mathrm{~mm}$ in diameter. Samples were fully submerged in water at room temperature (see the Supporting Information, Figure S2B). Hydrostatic pressure $(10,20,30,60$, and $100 \mathrm{mmHg})$ was generated using a $10 \mathrm{~L}$ tank filled with water and placed at different heights. A programmable PHD 2000 Harvard Apparatus syringe pump with a $60 \mathrm{~mL}$ syringe was used to generate a constant flow $(10 \mathrm{~mL} /$ $\mathrm{min})$ in the circuit. Ten images of the entire vessel were captured at each pressure value using a Canon EF $50 \mathrm{~mm}$ f/2.5 Compact Macro Lens. The pressure signal was acquired with a SPR-320 Mikro-Tip pressure catheter (Millar) equipped with a PCU-2000 pressure control unit (Millar) and recorded with LabVIEW. Data postprocessing was performed by a custom-made script developed in Matlab where vessel diameters were calculated by digital image analysis and related to the correspondent pressure value. Global compliance ${ }^{25}$ was calculated using

$$
C=\frac{\left(\mathrm{OD}_{90}-\mathrm{OD}_{60}\right) / \mathrm{OD}_{60}}{P_{90}-P_{60}}
$$

where $\mathrm{OD}$ and $P$ are the vessel outer diameter and pressure, respectively.

Polymer Degradation. Polymer films $(150 \pm 50 \mu$ m thickness, $n$ $=4)$ of each type were weighed $\left(W_{0}\right)$ and then placed in $10 \mathrm{~mL}$ of DPBS or $100 \mathrm{U}$ lipase/DPBS mixed solution at $37^{\circ} \mathrm{C}$ for hydrolytic or enzymatic degradation measurements, respectively. At each time point, the sample was washed using deionized water, dried in a vacuum oven at $60{ }^{\circ} \mathrm{C}$ for 3 days, and then weighed $\left(W_{1}\right)$. For the enzymatic degradation measurement, the fresh lipase/DPBS solution was exchanged every 3 days. At predetermined time points, the sample was rinsed $3 \times$ using deionized water and then dried in a vacuum oven at $60{ }^{\circ} \mathrm{C}$ for 3 days followed by weighing $\left(W_{1}\right)$. The mass remaining was calculated as $W_{1} / W_{0} \times 100 \%$. 


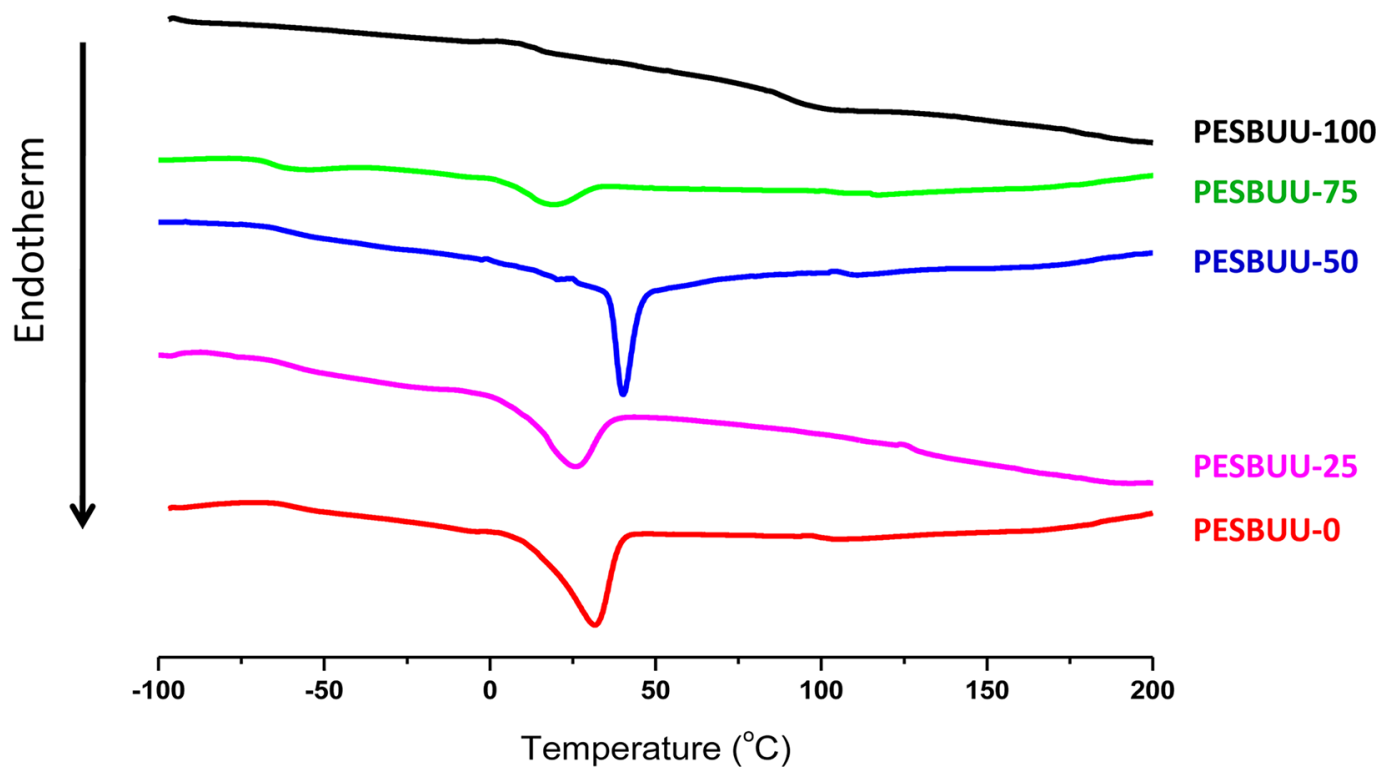

Figure 3. Differential scanning calorimetry spectra of PESBUUs.

Table 1. Thermal and Mechanical Properties of PESBUUs ${ }^{a}$

\begin{tabular}{|c|c|c|c|c|c|c|c|c|c|c|}
\hline \multirow[b]{2}{*}{ sample } & \multirow[b]{2}{*}{$T_{\mathrm{g}}\left({ }^{\circ} \mathrm{C}\right)$} & \multirow{2}{*}{$\begin{array}{c}T_{\mathrm{m}} \\
\left({ }^{\circ} \mathrm{C}\right)\end{array}$} & \multirow{2}{*}{$\begin{array}{c}X_{\mathrm{c}} b \\
(\%)^{b}\end{array}$} & \multicolumn{2}{|c|}{ tensile strength $(\mathrm{MPa})$} & \multicolumn{2}{|c|}{ strain at breaking (\%) } & \multicolumn{2}{|c|}{ initial modulus ( $\mathrm{MPa})$} & \multirow{2}{*}{$\begin{array}{l}\text { inherent viscosity } \\
(\mathrm{dL} / \mathrm{g})\end{array}$} \\
\hline & & & & dry & wet & dry & wet & dry & wet & \\
\hline PESBUU-0 & -58 & 32 & 32 & $37 \pm 3^{d}$ & $39 \pm 5^{d, e}$ & $1001 \pm 92^{f}$ & $1017 \pm 112^{e}$ & $26 \pm 2^{c}$ & $22 \pm 3^{d}$ & 1.24 \\
\hline PESBUU-25 & -58 & 26 & 23 & $43 \pm 7^{d, e}$ & $41 \pm 5^{e}$ & $825 \pm 68^{e}$ & $873 \pm 54^{d}$ & $22 \pm 5^{c}$ & $16 \pm 2^{c}$ & 1.23 \\
\hline PESBUU-50 & -58 & 40 & 34 & $48 \pm 7^{e}$ & $33 \pm 4^{d, g}$ & $851 \pm 78^{e}$ & $749 \pm 50^{d, g}$ & $24 \pm 4^{c}$ & $15 \pm 2^{c, g}$ & 1.08 \\
\hline PESBUU-75 & -66 & 19 & 10 & $42 \pm 6^{d, e}$ & $15 \pm 1^{c, g}$ & $629 \pm 47^{d}$ & $565 \pm 36^{c, g}$ & $90 \pm 20^{d}$ & $16 \pm 3^{c, g}$ & 0.80 \\
\hline PESBUU-100 & 16,97 & & & $21 \pm 3^{c}$ & & $85 \pm 16^{c}$ & & $265 \pm 50^{e}$ & & 0.48 \\
\hline
\end{tabular}

${ }^{a}$ Statistical comparisons of properties were made between PESBUU types under dry and wet conditions, respectively. ${ }^{b} \mathrm{Crystallinity}\left(X_{\mathrm{c}}\right) \%=100 \times$ $\Delta H / \Delta H_{100 \%}$, where $\Delta H 100 \%$ is the heat fusion of a perfect crystal of PCL, which was taken as $136 \mathrm{~J} / \mathrm{g}^{47,48} c, d, e$, and ${ }^{f}$ denote statistically distinct groups for each property and condition. ${ }^{g}$ denotes a statistical difference in comparing between dry and wet conditions within a given PESBUU type.

Protein Adsorption (Ovine Fibrinogen As a Model Protein). Surface protein adsorption on the PESBUU films was assessed by a microbicinchoninic acid (BCA) assay. ${ }^{30}$ Ovine fibrinogen (Sigma) solution was prepared in DPBS at a concentration $0.03 \mathrm{~g} / \mathrm{dL}$. The samples were immersed in the fibrinogen solution at $37^{\circ} \mathrm{C}$ for $3 \mathrm{~h}$. The samples were washed with DPBS, and then the absorbed protein was detached in $1 \mathrm{wt} \%$ aqueous solution of sodium dodecyl sulfate solution by shaking for $3 \mathrm{~h}$ at room temperature to detach the adsorbed protein on the surface. ${ }^{30}$ A protein analysis kit (QuantiproMicro BCA kit, Sigma-Aldrich, St. Louis, MO) based on the BCA method was utilized to quantify adsorbed fibrinogen. The mean value of fibrinogen adsorption from three independent samples, each measured in triplicate, was determined.

Ovine Blood Contact Test (with/without degradation). Whole ovine blood was collected by jugular venipuncture. NIH guidelines for the care and use of laboratory animals were observed, and all animal procedures were approved by the Institutional Animal Care and Use Committee (IACUC) at the University of Pittsburgh. In vitro thrombotic deposition on the polymer samples was assessed by a simple rocking test ${ }^{13}$ with heparinized ovine blood (heparin $3.0 \mathrm{U} / \mathrm{mL}$ added as a minimum anticoagulant for the test). Also polymer samples exposed to the enzymatic degradation test for $2 \mathrm{wk}$ and 2 mo were evaluated to investigate changes in thrombogenicity during the polymer degradation that might indicate a risk for late thrombosis after material implantation. Each polymer membrane type was cut into $10 \mathrm{~mm}$ diameter disks, sterilized with ethanol and placed in a test tube (BD Vacutainer, with no additives). The test tube was filled with $4 \mathrm{~mL}$ of ovine blood and gently rocked for $2 \mathrm{~h}$ at $37{ }^{\circ} \mathrm{C}$ on a hematology mixer (Fisher Scientific, Pittsburgh, PA). After ovine blood contact, the polymer membrane surfaces were rinsed with DPBS (10 times) to remove any nonadherent blood contents. Samples were then immersed in a $2.5 \%$ glutaraldehyde solution of DPBS for $2 \mathrm{~h}$ at 4 ${ }^{\circ} \mathrm{C}$ to fix the surface adherent platelets and then serially dehydrated with solutions of increasing ethanol content. Each sample surface was observed by scanning electron microscopy (SEM; JSM-6330F, JEOL USA, Inc., Peabody, MA) after sputter coating with gold/palladium. Deposited platelets on each surface were quantified by a lactate dehydrogenase (LDH) assay ${ }^{13}$ with an LDH Cytotoxicity Detection Kit (Clontech Laboratories, Inc. Mountain View, CA). Four samples for each group were performed.

Statistical Analyses. The results are presented as mean \pm standard deviation (SD). Data were analyzed by one-way ANOVA followed by a post hoc Neuman-Keuls testing. Significant differences were considered to exist at $p<0.05$.

\section{RESULTS}

Polymer Characterization. Typical ${ }^{1} \mathrm{H}$ NMR spectra of PESBUU-0, PESBUU-50 and PESBUU-100 are shown in Figure 2. PESBUU-0 synthesized using only PCL-diol shows typical peaks at $1.3-1.5 \mathrm{ppm}\left(\mathrm{CH}_{2}\right), 2.25 \mathrm{ppm}\left(\mathrm{OCCH}_{2}\right)$ and 3.95 ppm $\left(\mathrm{COCH}_{2}\right)$ originating from the PCL (red arrows). PESBUU-50 has the same peaks of the PCL as well as three new peaks at $0.85 \mathrm{ppm}\left(\mathrm{CH}_{2} \mathrm{CH}_{\underline{3}}\right), 2.05 \mathrm{ppm}\left(\mathrm{CH}_{2} \mathrm{CH}_{2} \mathrm{SO}_{3}\right)$, $2.90 \mathrm{ppm}\left(\mathrm{C}_{2} \mathrm{SO}_{3}\right), 3.55 \mathrm{ppm}\left(\mathrm{NC}_{2}\right)$ and $4.35 \mathrm{ppm}$ $\left(\mathrm{CH}_{2} \mathrm{O}\right)$ from the SB (blue arrows). The peaks from SB were more apparent in PESBUU-100 which was synthesized with SB-diol only, whereas the peaks from PCL disappeared. 
(A)

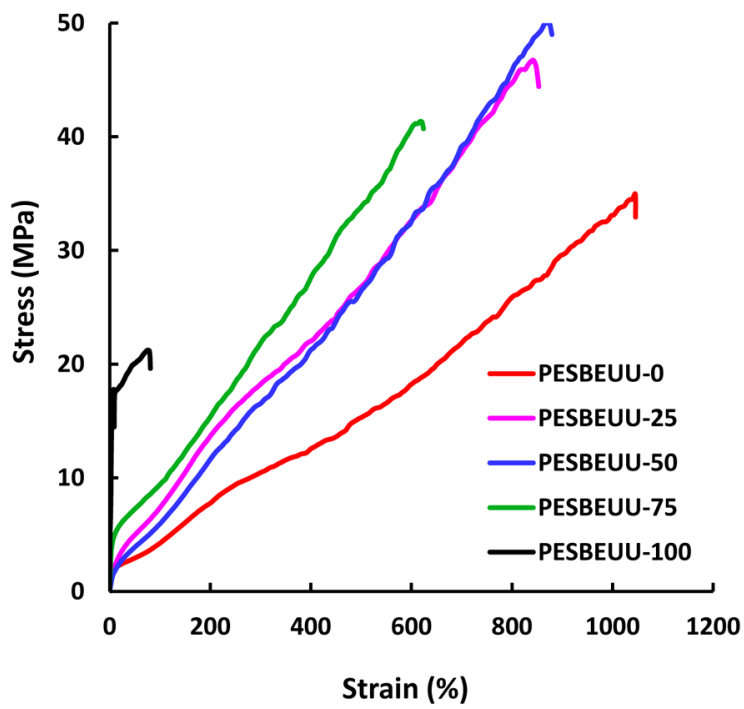

(B)

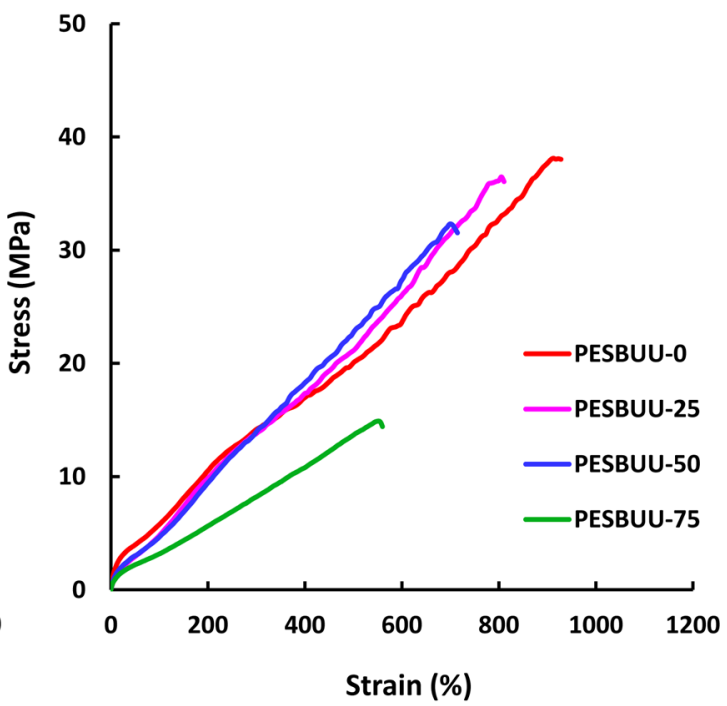

Figure 4. Typical stress-strain curves of cast PESBUU films measured under (A) dry and (B) wet conditions. PESBEUU-100 was too weak to assess under wet conditions.

(A)

PESBUU-0

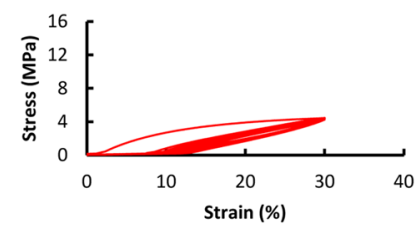

PESBUU-25

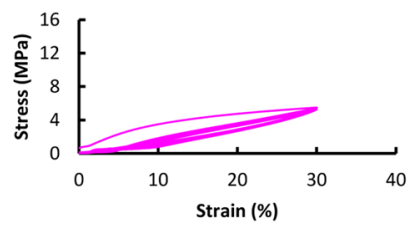

PESBUU-75

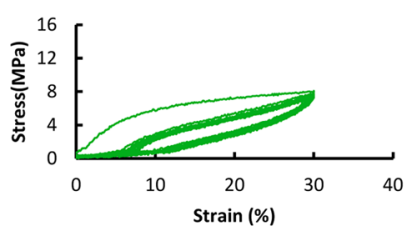

PESBUU-50

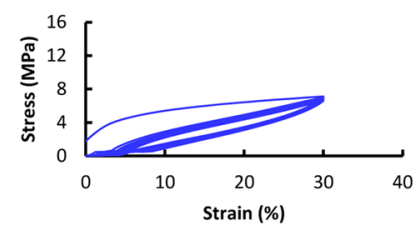

PESBUU-100

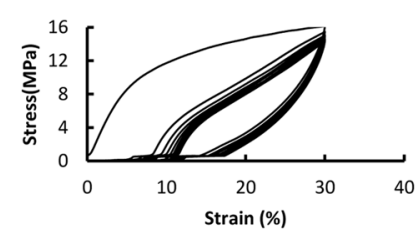

(B)

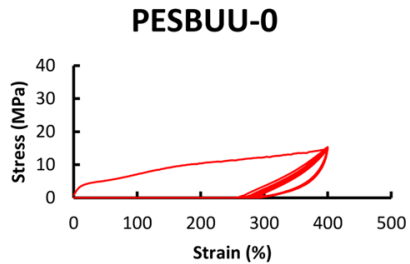

PESBUU-25

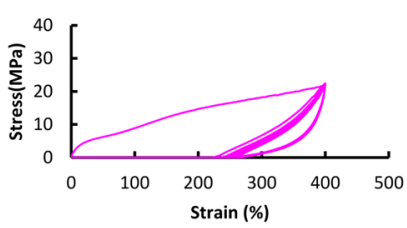

PESBUU-75

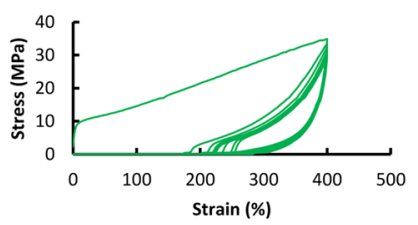

PESBUU-50

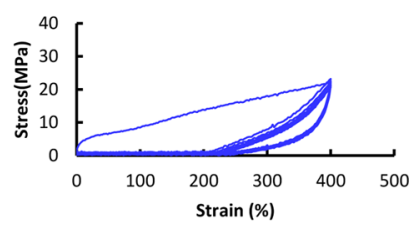

PESBUU-100

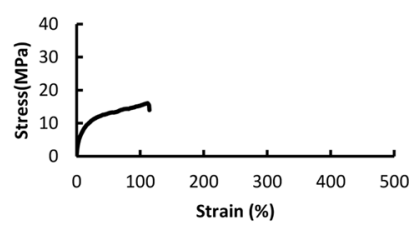

Figure 5. Cyclic tensile curves at (A) $30 \%$ and (B) $400 \%$ strain for PESBUUs.

Thermal properties are presented in Figure 3. The PESBUUs showed melting temperatures in the $25-40^{\circ}$ range with the exception of PESBUU-100, which lacked PCL. The glass transition temperature $\left(T_{\mathrm{g}}\right)$ for all polymers was lower than $-50{ }^{\circ} \mathrm{C}$, again with the exception of PESBUU-100 where no $T_{\mathrm{m}}$ could be clearly determined in the range of that seen for the other polymers (Table 1) and where there were two apparent $T_{\mathrm{g}} \mathrm{s}$ values were seen.

Mechanical Properties. Representative stress-strain curves for the PESBUUs in dry and wet conditions are shown in Figure 4 and the calculated mechanical parameters are summarized in Table 1 . The mechanical properties were seen to vary with the relative portions of $\mathrm{SB}$ and PCL in the
PESBUUs. Under dry conditions the PESBUU-50 had a statistically higher maximum tensile strength compared to the PESBUU-0 control synthesized from only PCL-diol, although PESBUU-25 and PESBUU-75 did not show significant differences with PESBUU-0. The strain at break of the PESBUUs decreased with increasing of SB content. The PESBUU-75 exhibited a significantly higher initial modulus compared to PESBUU-0, PESBUU-25, and PESBUU-50, whereas PESBUU-100 synthesized from only SB-diol was significantly stiffer than all of the other PESBUUs polymers with the lowest strain at break $(85 \pm 16 \%)$.

The mechanical properties for the wet polymers showed differences for the higher SB content polymers (Table 1). 
Whereas PESBUU-0 and PESBUU-25 did not exhibit significant differences compared to the dry state, the properties of PESBUU-50 and PESBUU-75 were decreased. However, the PESBUU-50 polymer still had a similar tensile strength (33 \pm 4 $\mathrm{MPa}$ ) with the PESBUU-0 and the strain at break was $749 \pm$ $50 \%$ in the wet condition. The mechanical properties of PESBUU-100 could not be measured because the polymer became weak and too soft to measure in the wet condition.

Figure 5 shows the results of cyclic tensile testing with a maximum strain of $30 \%$ (Figure 5A) and $400 \%$ (Figure 5B). All of the PESBUU polymers exhibited large hysteresis loops in the first cycle, followed by much smaller hysteresis loops in the next nine cycles. With a maximum strain of $30 \%$, the majority of samples showed a 5-10\% unrecoverable deformation, whereas PESBUU-100 had a slightly higher set than the others. The hysteresis loops of the next nine cycles were increased gradually with increasing SB content, and the PESBUU-100 showed clearly different behavior in this regard than the PESBUU-0. At a maximum strain of $400 \%$, the unrecoverable deformations were much higher $(200-250 \%)$ for all PESBUU samples compared to that seen with $30 \%$ maximum strain, but the trend in the hysteresis loops was similar to that observed at $30 \%$ strain. The PESBUU-100 that could not elongate to $400 \%$, and thus cyclic testing at this high strain was not possible.

The mechanical properties for electrospun sheets and conduits of PESBUU-0, PESBUU-50 and PESBUU-75 under wet conditions are summarized in Table 2. The electrospun

Table 2. Mechanical Properties of Electrospun PESBUU Sheets under Wet Conditions

$\begin{array}{ccccc}\text { sample } & \begin{array}{c}\text { tensile } \\ \text { strength } \\ (\mathrm{MPa})\end{array} & \begin{array}{c}\text { strain at } \\ \text { breaking } \\ (\%)\end{array} & \begin{array}{c}\text { initial } \\ \text { modulus } \\ (\mathrm{MPa})\end{array} & \begin{array}{c}\text { compliance } \\ 10^{-4} \mathrm{~mm} \\ \left.\mathrm{Hg}^{-1}\right)^{a}\end{array} \\ \text { PESBUU-0 } & 9.5 \pm 2.3^{c} & 278 \pm 27^{c} & 4.7 \pm 1.7^{b} & 7.2 \pm 2.0^{b} \\ \text { PESBUU-50 } & 7.9 \pm 1.8^{c} & 301 \pm 23^{c} & 2.5 \pm 0.7^{b} & 4.0 \pm 2.4^{b} \\ \text { PESBUU-75 } & 4.8 \pm 1.2^{b} & 163 \pm 29^{b} & 3.5 \pm 1.1^{b} & 5.3 \pm 1.0^{b}\end{array}$

${ }^{a}$ Compliance values were calculated between 60 and $90 \mathrm{mmHg}^{b}$ and ${ }^{c}$ denote statistically distinct groups for each property and condition.

sheets showed 4.8-9.5 MPa tensile strength, 163-301\% strain at break, and 2.5-4.7 MPa initial modulus. The PESBUU-50 electrospun sheets still exhibited similar mechanical properties with PESBUU-0 control, although the PESBUU-75 showed significantly decreased tensile strength and strain at break. Compliance of the PESBUU-50 conduit (1.0 mm diam) calculated between 60 and $90 \mathrm{mmHg}$ of hydrostatic pressure was $4.0 \pm 2.4 \times 10^{-4} \mathrm{~mm} \mathrm{Hg}^{-1}$ (Table 2).

Water Absorption and Protein Adsorption. Water absorption for each of the PESBUUs films is shown in Figure 6A. Water absorption gradually increased with increasing SB content, and the wt $\%$ of absorbed water reached up to $28.1 \%$ for PESBUU-100. In contrast, the absorption for PESBUU-0 and PESBUU-50 were 1.6 and $5.7 \%$, respectively.

The amount of adsorbed ovine fibrinogen on the PESBUU polymer films is summarized in Figure 6B. Fibrinogen adsorption gradually decreased with increasing SB content in the PESBUU polymers, although between the two relatively high SB content and the two relatively low SB content polymers the adsorption was statistically comparable.

Polymer Degradation. The polymer degradation behavior in lipase solution measured for 3 months is shown in Figure 7.

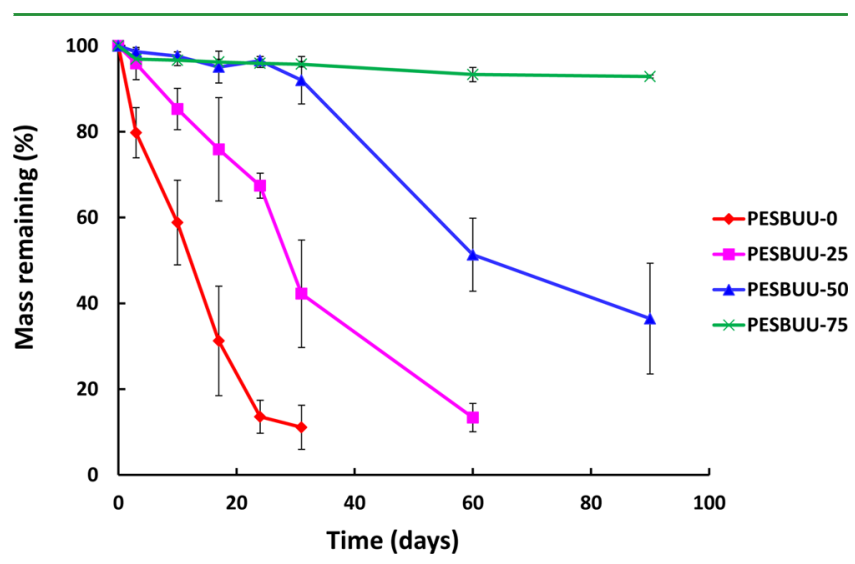

Figure 7. Mass remaining for PESBUUs films in $100 \mathrm{U} / \mathrm{mL}$ lipase solution of PBS at $37{ }^{\circ} \mathrm{C}$.

The PESBUU-0 control experienced $89 \%$ weight loss in one month. However, the amount of mass loss was reduced with increasing $\mathrm{SB}$ content such that PESBUU-25 required an additional month to reach $87 \%$ mass loss, and the PESBUU-50 showed $64 \%$ weight loss at 3 months. PESBUU-75 only lost $7 \%$
(A)

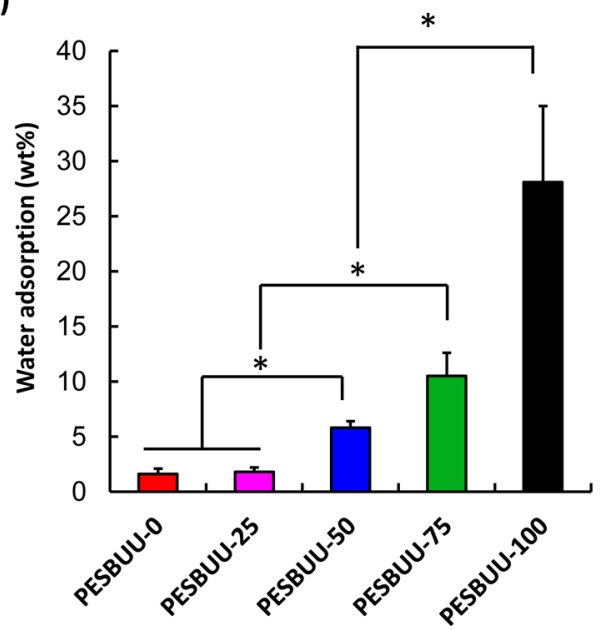

(B)

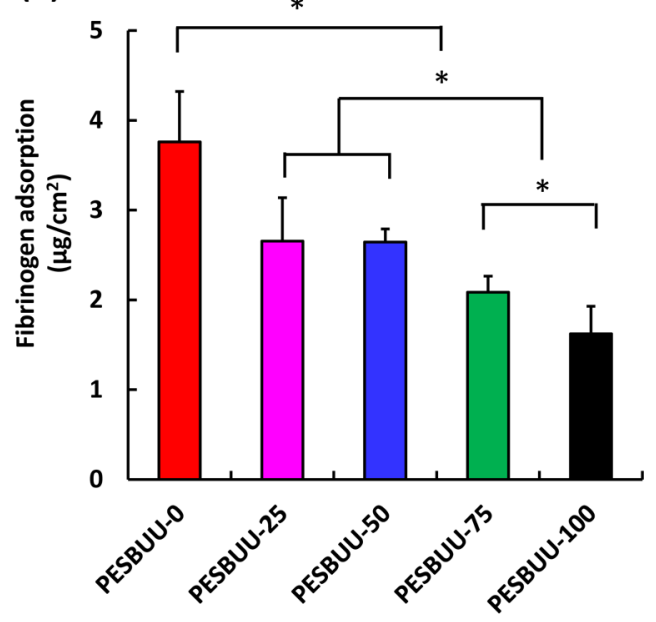

Figure 6. (A) Water absorption after immersion in DPBS for $24 \mathrm{~h}$ at $37^{\circ} \mathrm{C}$ and (B) ovine fibrinogen adsorption on PESBUUs films. 


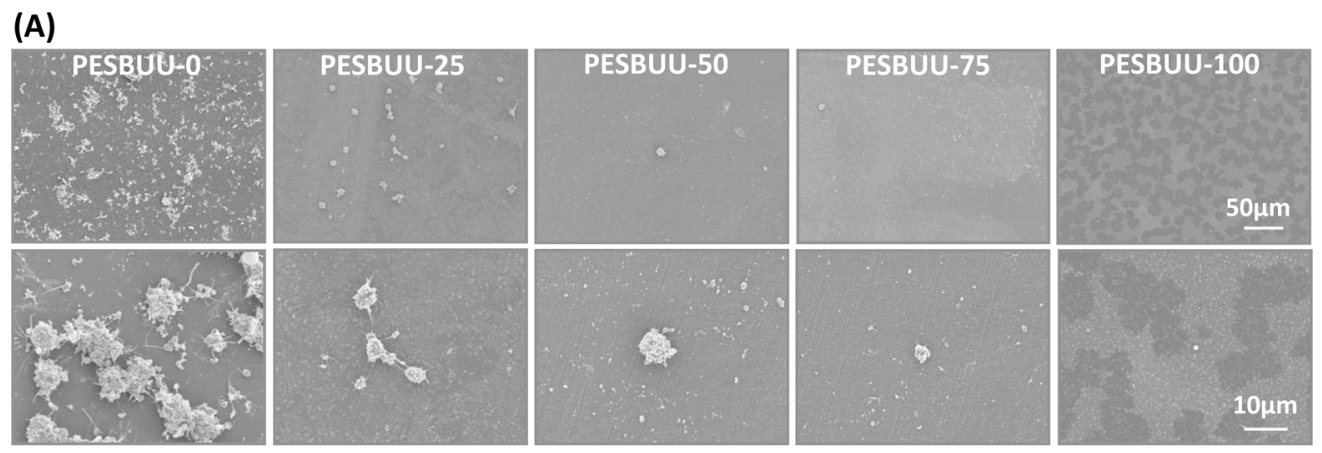

(B)

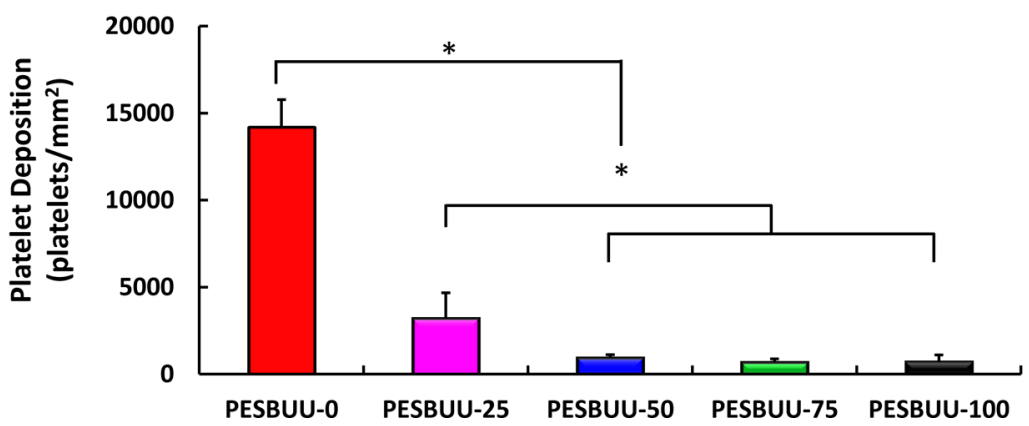

Figure 8. Ovine blood platelet deposition on PESBUU films (A) observed by scanning electron microscopy after contact with ovine blood (heparin $3 \mathrm{U} / \mathrm{mL}$ ) for $2 \mathrm{~h}$ at $37^{\circ} \mathrm{C}$. (B) Platelet deposition on polymer films as quantified by LDH assay.

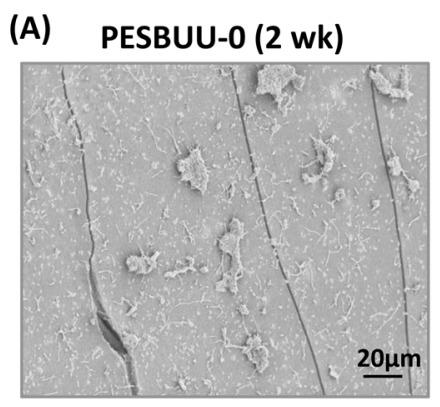

(B)

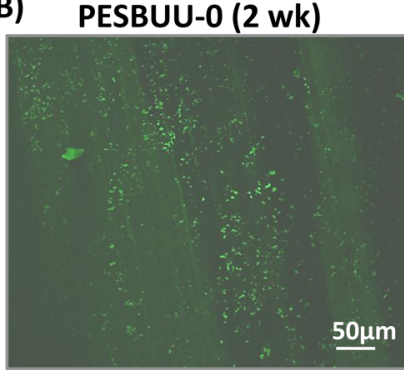

PESBUU-50 (2 mo)

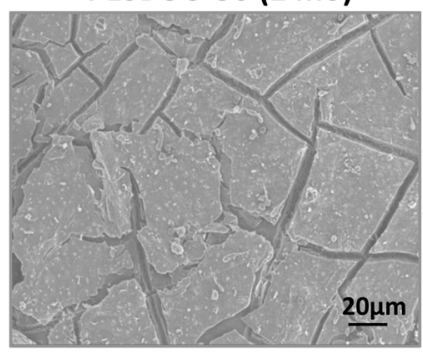

PESBUU-50 (2 mo)

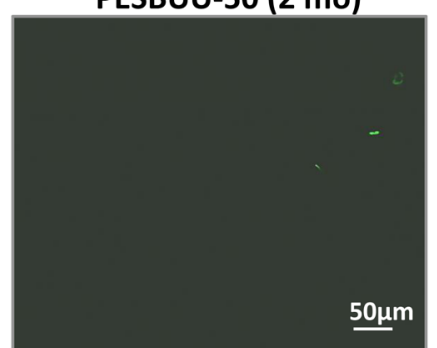

PESBUU-75 (2 mo)

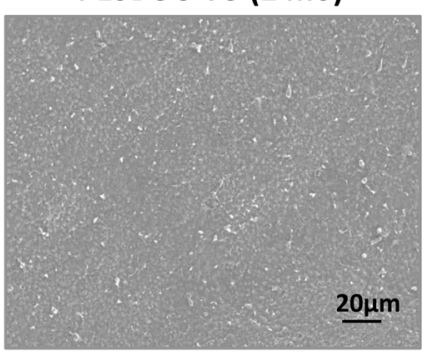

PESBUU-75 (2 mo)

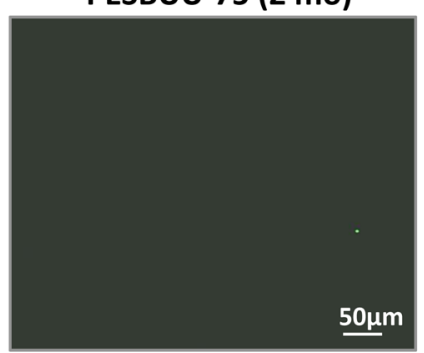

Figure 9. (A) Scanning micrographs and (B) confocal micrographs of partially degraded PESBUU-0 (2 wk), PESBUU-50 (2 mo), and PESBUU-75 $(2 \mathrm{mo})$ after contact with ovine blood (heparin $3 \mathrm{U} / \mathrm{mL}$ ) for $2 \mathrm{~h}$ (deposited platelets were stained with fluorescent mepacrine).

mass at 3 months, and PESBUU-100 films fractured into small pieces during the test period, making accurate mass measurements impractical, although the pieces did not appear to obviously be losing mass. In DPBS alone, without lipase, polymer degradation was much reduced and did not vary between polymer types (see the Supporting Information, Figure S3).

Ovine Platelet Deposition and Electrospun Scaffolds. Platelet deposition from whole ovine blood onto PESBUU films observed by scanning electron microscopy after blood contact for $2 \mathrm{~h}$ is shown in Figure 8A. The PESBUU-0 surfaces showed moderate levels of platelet deposition and the deposited platelet aggregates were observed over the blood contacting area. Platelet deposition decreased with increasing SB content. Deposited platelets were sparse on the PESBUU50 films and finding deposited platelets on PESBUU-75 and PESBUU-100 surfaces was difficult. Quantification of platelet deposition using an LDH assay (Figure 8B) confirmed the visual results, with platelet deposition reduced on PESBUU-25 and further decreased on PESBUU-50, PESBUU-75 and PESBUU-100 polymers, where the average reduction versus PESBUU-0 reached up to $95 \%$.

To qualitatively investigate the retention of the nonthrombogenic character for degrading PESBUU samples, 
(A)

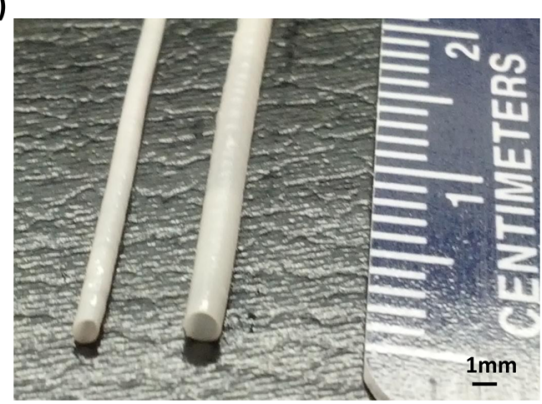

(B)

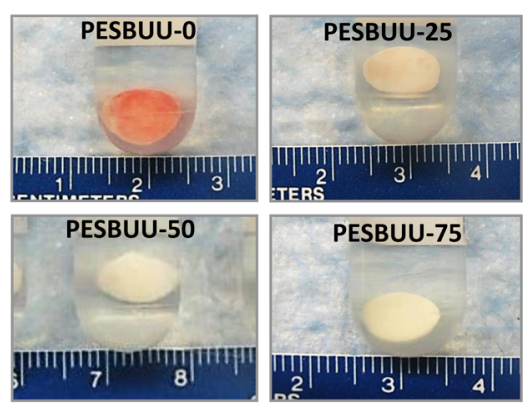

PESBUU-75
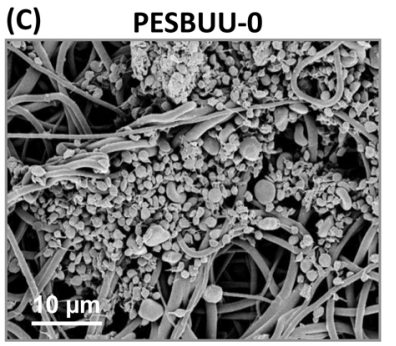

PESBUU-50
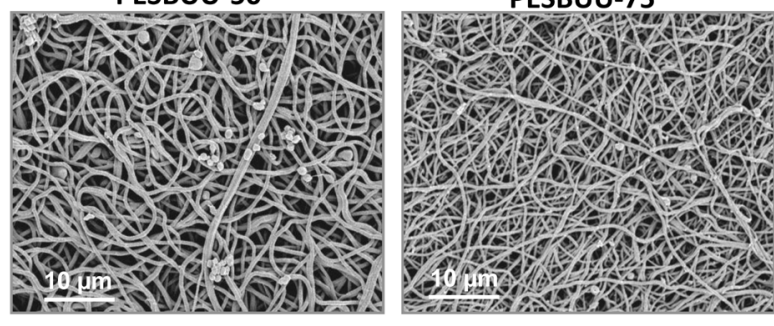

Figure 10. (A) Electrospun conduits of PESBUU-50 with different diameters (1.0 mm, left; and $1.6 \mathrm{~mm}$, right) (B) macroscopic and (C) scanning electron micrographs of electrospun PESBUU sheets after contact with ovine blood (heparin $3 \mathrm{U} / \mathrm{mL}$ ) for $3 \mathrm{~h}$ at $37^{\circ} \mathrm{C}$.

ovine blood was contacted with PESBUU samples after enzymatic degradation testing (Figure 9). The PESBUU-0 obtained after 2 weeks (wk) showed scattered deposited platelets and platelet aggregates (Figure 9). However, it was hard to find deposited or aggregated platelets on the PESBUU50 and PESBUU-75 polymer surfaces that had been partially degraded over 2 months (mo). Confocal micrographs (Figure 9B) obtained after fluorescently staining deposited platelets showed that there was rare platelet deposition on the PESBUU50, and the PESBUU-75 also did not show any notable platelet deposition.

PESBUU was readily processable with electrospinning, into both sheet and small conduit formats. The latter conduits (Figure 10A) were generated at a size compatible with large rodent implantation. The former were used to further explore thrombogenicity of the fibrous polymer surface. Macroscopic and electron micrographs of electrospun PESBUU sheets after contact with ovine blood (heparin $3 \mathrm{U} / \mathrm{mL}$ ) for $3 \mathrm{~h}$ are shown in Figure 10B, C. After the ovine blood contact, thrombotic deposition was apparent on the PESBUU-0 electrospun sheet at both the macroscopic and microscopic level, with the latter exhibiting deposited platelet aggregates with some entrapped erythrocytes. Macroscopic thrombotic deposition was visibly decreased on the PESBUU-25 and not noted on the electrospun scaffolds of PESBUU-50 and PESUU-75 (Figure 10B), which corresponded with micrographs showing sparsely deposited platelets on PESBUU-50 and PESBUU-75 electrospun scaffolds (Figure 10C).

\section{DISCUSSION}

Poly(ester urethane)ureas combined with zwitterionic PCcontaining polymers by either blending or surface grafting have previously been shown to have reduced thrombogenicity both in vitro and in vivo. ${ }^{25-27}$ However, these approaches are limited by the amount of PC-containing polymer that might be blended and still preserve mechanical properties, the elution of PC-containing blended polymer as the primary polymer component degrades, and concerns of underlying substrate exposure at later times with loss of surface bound moieties from polymers with zwitterion surface-grafting. Thus, in this study, a hydrolytically labile polyurethane with variable SB content as part of the polymer backbone was designed that would putatively be a fully degradable nonthrombogenic elastomer with physical and nonthrombogenic properties being tunable with the SB composition.

Cao et al. ${ }^{17}$ have reported the synthesis of an SB-containing polycaprolactone (PCL-APS) by mixing a similar SB-diol and an $\varepsilon$-caprolactone (CL) monomer to initiate ring-opening polymerization. The molecular weight of PCL-APS was changed by varying the ratio of monomer (CL) to the initiator (SB-diol) to alter the number of repeating CL units. Further, they also synthesized a polyurethane with $\mathrm{SB}$ by chain extension of the PCL-APS with hexamethylene diisocyanate. ${ }^{18}$ However, this approach varies from the current report in that control of SB content in the polyurethane was fixed at one SB per diol, whereas here the ratio of PCL-diol and SB diol could be varied to tune the physical properties of the PESBUUs in addition to the degradation and thrombogenic behavior. Further, with the current synthetic approach, the SB portion could be regarded as part of the hard-segment in the PESBUUs.

With increased SB content, PCL soft segment content decreased and thus the portion of hard segments was increased. The initial modulus of PESBUU-75 in the dry state (90 MPa) was significantly increased versus PESBUUs with lower SB content, and the PESBUU-100 synthesized from only SB-diol was more stiff (265 MPa initial modulus), with the lowest breaking strain. This effect could be attributed to the greater hard segment content for these PESBUUs with limited PCL soft segment content. However, both the initial modulus and tensile strength of PESBUU-75 were dramatically decreased (16 and $15 \mathrm{MPa}$, respectively) in wet conditions. The large changes in wet conditions compared with lower SB content polymers (PESBUU-25 and PESBUU-50) might be related to the increased swelling of the high SB-content PESBUUs and chain rearrangement related to the zwitterionic components. ${ }^{18}$

Degradation of the synthesized polymers in DPBS solution alone was markedly slower and did not vary notably among the polymer types compared to when lipase was present. PESBUU 
degradation is presumably due to ester bond cleavage of the soft segment PCL, relatively slowly degrading polyester due to its hydrophobicity and crystallinity. Hydrogen bonding in the polyurethane may also reduce the hydrolytic degradation rate of the PCL soft segments. Enzymatic degradation of PESBUUs in lipase obviously accelerated polymer degradation, suggesting that the polyurethanes would degrade faster in vivo than in vitro, as has been previously observed with similar polyurethanes. ${ }^{31,32}$ On the other hand, the difference in the polymer degradation profiles in lipase (Figure 7) was pronounced, with SB moiety introduction significantly reducing degradation. An increase in the SB portion (PCL portion decrease) led to slower degradation such that the mass loss of PESBUU-75 was less than $10 \%$ during the 3 month in a lipase solution. The decreased enzymatic degradation with increased SB might be a result of increased hard segment content, ${ }^{31,33}$ and the concomitant decrease in hydrolytically labile polyester with the decrease in the soft segment. One further consideration is that as the zwitterion content increases, resistance to protein, and thus enzyme, interactions on the PESBUU polymer surfaces might reduce the ability of these proteins to catalzye the degradation reaction. ${ }^{34}$ Additionally, the crystallinity of PCL blocks together with changes in the SB portions (hardsegments) of PESBUUs might also be important to explain the polymer degradation and the mechanical properties of the electrospun conduits and films. Further detailed studies might be pursued to define the relationship of the polymer degradation and mechanical properties with the polymer crystallinity using multiple methods (e.g., X-ray scattering and DSC).

Several previous studies have shown reduced protein adsorption in the presence of zwitterionic moieties, and the association of this low protein adsorption with decreased platelet deposition. ${ }^{11-13,18,24,30}$ PESBUU polymers containing SB moieties had lower fibrinogen adsorption on the surfaces compared to the control, and the amount of adsorbed fibrinogen decreased with increasing the SB content. Platelet deposition was markedly decreased in general correspondence with the SB content of the polymers. While it has been shown that the activity of fibrinogen on the surface in terms of platelet binding can be independent of the total amount of fibrinogen adsorbed, ${ }^{35}$ the data of this report suggest that reductions in protein adsorption might be responsible for the reduced platelet deposition. Further, it was shown that these biodegradable SB-bearing polyurethanes maintained their nonthrombogenic properties after periods of degradation, which might not be expected with surface modification or grafting approaches on degradable polymers. This sustained thromboresistance is a relevant property for applications where late thrombosis ${ }^{36,37}$ might be a concern.

In addition to the characterization of PESBUU polymer films, fibrous sheets, and small diameter conduits were fabricated by electrospinning. These processed surfaces exhibited significantly reduced thrombotic deposition with increasing SB content (Figure 10B, C) and suggested the feasibility of applying this material in the creation of a tissue engineered small diameter vascular graft (Figure 10A). As expected from the study of the polymer films, the PESBUU-50 electrospun sheet also was resistant to platelet deposition, and had mechanical properties similar to PESBUU-0 under wet conditions and at $37^{\circ} \mathrm{C}$, that has previously been evaluated as a vascular replacement graft in rodents. ${ }^{25,26}$ PESBUU-0 exhibited values of both initial elastic moduli and global compliance comparable to those reported in previously, ${ }^{25}$ and the compliance of PESBUU 50 and PESBUU 75 showed global compliance values in a range similar to native blood vessels (4$\left.5 \times 10^{-4} \mathrm{~mm} \mathrm{Hg}^{-1}\right){ }^{38}$ Further studies might be directed to optimize the electrospinning process for vascular graft fabrication depending on specific PESBUU polymer characteristics. As can be seen in the different intrinsic viscosities of the different PESBUU polymer solutions (Table 1), variable incorporation of the zwitterionic SB moieties in polymer altered the solution behavior and thus would impact the processing parameters to achieve eletrospun scaffolds with desired morphological characteristics such as fiber diameter, fiber density, and porosity.

This report is limited in several aspects, particularly with respect to the appropriateness of the materials for the speculated cardiovascular applications. The assessment of nonthrombogenic behavior has been done with an acute, in vitro, ovine blood contacting test with anticoagulants present. Although this test provided relative discrimination between polymer types, the lack of thrombotic deposition here might not translate to various clinical applications directly. Also along these lines, the in vitro degradation, even with enzymes, does not capture the complex in vivo situation where phagocytic leukocyte involvement results in an array of enzymes and other factors (e.g., strong oxidants) that would generally be expected to accelerate degradation and the loss of mechanical properties. This report did not investigate the loss of mechanical properties with degradation, and this is a relevant feature for load-bearing scaffolds placed in many applications. For instance, early loss of mechanical properties might lead to aneurysmal dilatation of a vascular scaffold if not enough tissue ingrowth has occurred to compensate. In that this report describes the method for the generation of a family of degradable nonthrombogenic polyurethanes, the means to tune the selection of an appropriate polymer where trade offs are made between various physical and functional considerations is present. Furthermore, polymers similar to PESBUU-0 have been implanted in functional locations in small animal models and shown polymer degradation accompanied by tissue remodeling appropriate to maintain mechanical viability or mechanical effect. $^{39-41}$

On the basis of the limited assessments of this manuscript, the PESBUU-50 appears to provide the most attractive compromise between high strength (over $30 \mathrm{MPa}$ ) and strain (over $700 \%$ ) under both dry and wet conditions, processability and thromboresistance, although PESBUU-75 had somewhat similar behavior. Those polymers could be fabricated into electrospun conduits that qualitatively appeared mechanically appropriate for vascular scaffold applications. ${ }^{42-45}$ Furthermore, the fully degradable PESBUU elastomers might also find other suitable cardiovascular applications including coatings for a drug-eluting biodegradable stent ${ }^{46}$ as well as tissue engineered heart valves. ${ }^{47,48}$

\section{CONCLUSIONS}

A series of biodegradable and robust elastic polyurethanes were synthesized with $\mathrm{SBs}$ as part of the polymer backbone (PESBUUs), and where the SB content in the polymer could be easily tuned by altering the molar ratio of PCL and SB diol reactants. The mechanical and degradation properties were sensitive to the SB content, with enzymatic degradation being dependent on the PCL content. At higher zwitterionic molar ratios (PESBUU-50 and PESBUU-75) markedly reduced 
thrombotic deposition was observed both before and after substantial degradation. The mechanical and degradation properties as well as the acute in vitro thrombogenicity assessment suggest that these polymers would be appropriate for use in blood contacting applications where a degradable, elastomeric component with enduring thromboresistance is desired.

\section{ASSOCIATED CONTENT}

\section{(5) Supporting Information}

${ }^{1} \mathrm{H}$ NMR spectra of sulfobetaine-diol (SB-diol) (Figure S1), global mechanical compliance apparatus (Figure S2), and mass remaining for PESBUUs films in DPBS at $37{ }^{\circ} \mathrm{C}$ (Figure S3). This material is available free of charge via the Internet at http://pubs.acs.org.

\section{AUTHOR INFORMATION}

\section{Corresponding Author}

*E-mail: wagnerwr@upmc.edu. Phone: 001-412-624-5324. Fax: 001-412-624-5363.

\section{Present Address}

${ }^{\S}$ Y.H. is currently at Department of Bioengineering, University of Texas at Arlington, Arlington, TX 76019, United States.

\section{Author Contributions}

II The first and second authors contributed equally to this work.

\section{Notes}

The authors declare no competing financial interest.

\section{ACKNOWLEDGMENTS}

This research was supported by the National Science Foundation (ERC-RMB, Award NSF 0812348). The authors thank the Center for Biological Imaging (CBI) of the University of Pittsburgh for their kind assistance in obtaining the SEM images.

\section{REFERENCES}

(1) Sun, T.; Tan, H.; Han, D.; Fu, Q.; Jiang, L. No Platelet Can Adhere-Largely Improved Blood Compatibility on Nanostructured Superhydrophobic Surfaces. Small 2005, 1, 959-963.

(2) Xiang, T.; Yue, W. W.; Wang, R.; Liang, S.; Sun, S. D.; Zhao, C. S. Surface Hydrophilic Modification of Polyethersulfone Membranes by Surface-initiated ATRP with Enhanced Blood Compatibility. Colloids Surf., B 2013, 110, 15-21.

(3) Wang, L. R.; Qin, H.; Nie, S. Q.; Sun, S. D.; Ran, F.; Zhao, C. S. Direct Synthesis of Heparin-like Poly(ether sulfone) Polymer and Its Blood Compatibility. Acta Biomater. 2013, 9, 8851-8863.

(4) Pan, C. J.; Hou, Y. H.; Zhang, B. B.; Dong, Y. X.; Ding, H. Y. Blood Compatibility and Interaction with Endothelial Cells of Titanium Modified by Sequential Immobilization of Poly (ethylene glycol) and Heparin. J. Mater. Chem. B 2014, 2, 892-902.

(5) Sin, M. C.; Sun, Y. M.; Chang, Y. Zwitterionic-based Stainless Steel with Well-defined Polysulfobetaine Brushes for General Bioadhesive Control. ACS Appl. Mater. Interfaces 2014, 6, 861-873.

(6) Sin, M. C.; Chen, S. H.; Chang, Y. Hemocompatibility of Zwitterionic Interfaces and Membranes. Polym. J. 2014, 46, 436-443.

(7) Qiu, Y.; Holland, N. B.; Ruegsegger, M.; Marchant, R. E. Biomimetic Surface Engineering: Synthesis and Surface-induced Assembly of Novel Oligosaccharide Surfactant Polymers. In Supramolecular Structure in Confined Geometries; ACS Symposium Series; American Chemical Society: Washington, D.C., 1999; pp 180-193.

(8) Thasneem, Y. M.; Rekha, M. R.; Sajeesh, S.; Sharma, C. P. Biomimetic Mucin Modified PLGA Nanoparticles for Enhanced Blood Compatibility. J. Colloid Interface Sci. 2013, 409, 237-244.
(9) Holmlin, R. E.; Chen, X.; Chapman, R. G.; Takayama, S.; Whitesides, G. M. Zwitterionic SAMs That Resist Nonspecific Adsorption of Protein from Aqueous Buffer. Langmuir 2001, 28412850

(10) Kudaibergenov, S.; Jaeger, W.; Laschewsky, A. Polymeric Betaines: Synthesis, Characterization, and Application. Adv. Polym. Sci. 2006, 201, 157-224.

(11) Zhang, Z.; Chao, T.; Chen, S.; Jiang, S. Superlow Fouling Sulfobetaine and Carboxybetaine Polymers on Glass Slides. Langmuir 2006, 22, 10072-10077.

(12) Zhang, Z.; Zhang, M.; Chen, S.; Horbett, T. A.; Ratner, B. D.; Jiang, S. Blood Compatibility of Surfaces with Superlow Protein Adsorption. Biomaterials 2008, 29, 4285-4291.

(13) Ye, S. H.; Johnson, C. A., Jr; Woolley, J. R.; Murata, H.; Gamble, L. J.; Ishihara, K.; Wagner, W. R. Simple Surface Modification of a Titanium Alloy with Silanated Zwitterionic Phosphorylcholine or Sulfobetaine Modifiers to Reduce Thrombogenicity. Colloids Surf., B 2010, 79, 357-364.

(14) Kuo, W. H.; Wang, M. J.; Chien, H. W.; Wei, T. C.; Lee, C.; Tsai, W. B. Surface Modification with Poly(sulfobetaine methacrylateco-acrylic acid) To Reduce Fibrinogen Adsorption, Platelet adhesion, and Plasma Coagulation. Biomacromolecules 2011, 12, 4348-4356.

(15) Yuan, J.; Lin, S.; Shen, J. Enhanced Blood Compatibility of Polyurethane Functionalized with Sulfobetaine. Colloids Surf., B 2008, 66, 90-95.

(16) Smith, R. S.; Zhang, Z.; Bouchard, M.; Li, J.; Lapp, H. S.; Brotske, G. R.; Lucchino, D. L.; Weaver, D.; Roth, L. A.; Coury, A.; Biggerstaff, J.; Sukavaneshvar, S.; Langer, R.; Loose, C. Vascular Catheters with a Nonleaching Poly-sulfobetaine Surface Modification Reduce Thrombus Formation and Microbial Attachment. Sci. Transl. Med. 2012, 4, 153ra132.

(17) Cao, J.; Chen, Y. W.; Wang, X.; Luo, X. L. Enhancing Blood Compatibility of Biodegradable Polymers by Introducing Sulfobetaine. J. Biomed. Mater. Res., Part A 2011, 97, 472-479.

(18) Cao, J.; Yang, M.; Lu, A.; Zhai, S.; Chen, Y.; Luo, X. Polyurethanes Containing Zwitterionic Sulfobetaines and Their Molecular Chain Rearrangement in Water. J. Biomed. Mater. Res., Part A 2013, 101, 909-918.

(19) Santerre, J. P.; Woodhouse, K.; Laroche, G.; Labow, R. S. Understanding the Biodegradation of Polyurethanes: from Classical Implants to Tissue Engineering Materials. Biomaterials 2005, 26, $7457-7470$.

(20) Guelcher, S. A. Biodegradable Polyurethanes: Synthesis and Applications in Regenerative Medicine. Tissue Eng., Part B Rev. 2008, 14, 3-17.

(21) Guo, Q.; Knight, P. T.; Mather, P. T. Tailored Drug Release from Biodegradable Stent Coatings Based on Hybrid Polyurethanes. J. Controlled Release 2009, 137, 224-233.

(22) Bernacca, G. M.; Gulbransen, M. J.; Wilkinson, R.; Wheatley, D. J. In Vitro Blood Compatibility of Surface-modified Polyurethanes. Biomaterials 1998, 19, 1151-1165.

(23) Tan, D.; Zhang, X.; Li, J.; Tan, H.; Fu, Q. Modification of Poly(ether urethane) with Fluorinated Phosphorylcholine Polyurethane for Improvement of the Blood Compatibility. J. Biomed. Mater. Res., Part A 2011, 100A, 380-387.

(24) Taite, L. J.; Yang, P.; Jun, H. W.; West, J. L. Nitric Oxidereleasing Polyurethane-PEG Copolymer Containing the YIGSR Peptide Promotes Endothelialization with Decreased Platelet Adhesion. J. Biomed. Mater. Res. B 2008, 84, 108-116.

(25) Hong, Y.; Ye, S. H.; Nieponice, A.; Soletti, L.; Vorp, D. A.; Wagner, W. R. A Small Diameter, Fibrous Vascular Conduit Generated from a Poly(ester urethane) urea and Phospholipid Polymer Blend. Biomaterials 2009, 30, 2457-2467.

(26) Soletti, L.; Nieponice, A.; Hong, Y.; Ye, S. H.; Stankus, J. J.; Wagner, W. R; Vorp, D. A. In Vivo Performance of a Phospholipidcoated Bioerodable Elastomeric Graft for Small-diameter VascularA. J. Biomed. Mater. Res., Part A 2011, 96, 436-448.

(27) Hong, Y.; Ye, S. H.; Pelinescu, A. L.; Wagner, W. R. Synthesis, Characterization, and Paclitaxel Release from a Biodegradable, 
Elastomeric, Poly(ester urethane)urea Bearing Phosphorylcholine Groups for Reduced Thrombogenicity. Biomacromolecules 2012, 13, 3686-3694.

(28) Hong, Y.; Guan, J.; Fujimoto, K. L.; Hashizume, R.; Pelinescu, A. L.; Wagner, W. R. Tailoring the Degradation Kinetics of Poly(ester carbonate urethane)urea Thermoplastic Elastomers for Tissue Engineering Scaffolds. Biomaterials 2010, 31, 4249-4258.

(29) Ma, Z.; Hong, Y.; Nelson, D. M.; Pichamuthu, J. E.; Leeson, C. E.; Wagner, W. R. Biodegradable Polyurethane Ureas with Variable Polyester or Polycarbonate Soft Segments: Effects of Crystallinity, Molecular Weight, and Composition on Mechanical Properties. Biomacromolecules 2011, 12, 3265-3674.

(30) Ye, S. H.; Watanabe, J.; Iwasaki, Y.; Ishihara, K. Antifouling Blood Purification Membrane Composed of Cellulose Acetate and Phospholipid Polymer. Biomaterials 2003, 24, 43-52.

(31) Tang, Y. W.; Labow, R. S.; Santerre, J. P. Enzyme-induced Biodegradation of Polycarbonate Polyurethanes: Dependence on Hard-segment Concentration. J. Biomed. Mater. Res. 2001, 56, 516528.

(32) Tang, Y. W.; Labow, R. S.; Santerre, J. P. Enzyme Induced Biodegradation of Polycarbonate-polyurethanes: Dose Dependence Effect of Cholesterol Esterase. Biomaterials 2003, 24, 2003-2011.

(33) Mochizuki, M.; Mukai, K.; Yamada, K.; Ichise, N.; Murase, S.; Iwaya, Y. Structural Effects upon Enzymatic Hydrolysis of Poly(butylene succinate-co-ethylene succinate)s. Macromolecules 1997, 30, 7403-7407.

(34) Yu, B. Y.; Zheng, J.; Chang, Y.; Sin, M. C.; Chang, C. H.; Higuchi, A.; Sun, Y. M. Surface Zwitterionization of Titanium for a General Bio-Inert Control of Plasma Proteins, Blood Cells, Tissue Cells, and Bacteria. Langmuir 2014, 30, 7502-7512.

(35) Tsai, W. B.; Grunkemeier, J. M.; Horbett, T. A. Variations in the Ability of Adsorbed Fibrinogen to Mediate Platelet Adhesion to Polystyrene-based Materials: a Multivariate Statistical Analysis of Antibody Binding to the Platelet Binding Sites of Fibrinogen. J. Biomed. Mater. Res., Part A 2003, 67, 1255-1268.

(36) Pfisterer, M. E. Late Stent Thrombosis after Drug-Eluting Stent Implantation for Acute Myocardial Infarction: A New Red Flag Is Raised. Circulation 2008, 118, 1117-1119.

(37) Li, P.; Liu, J. P. Long-term Risk of Late and Very Late Stent Thrombosis in Patients Treated with Everolimus Against PaclitaxelEluting Stents: An Update Meta-analysis. Coron. Artery Dis. 2014, 25, 369-377.

(38) Catto, V.; Farè, S.; Freddi, G.; Tanzi, M. C. Vascular Tissue Engineering: Recent Advances in Small Diameter Blood Vessel Regeneration. ISRN Vasc. Med. 2014, 923030, 1-27.

(39) Fujimoto, K. L.; Guan, J.; Oshima, H.; Sakai, T.; Wagner, W. R. In Vivo Evaluation of a Porous, Elastic, Biodegradable Patch for Reconstructive Cardiac Procedures. Ann. Thorac. Surg. 2007, 83, 648654.

(40) Hong, Y.; Takanari, K.; Amoroso, N. J.; Hashizume, R.; Brennan-Pierce, E. P.; Freund, J. M.; Badylak, S. F.; Wagner, W. R. An Elastomeric Patch Electrospun from a Blended Solution of Dermal Extracellular Matrix and Biodegradable Polyurethane for Rat Abdominal Wall Repair. Tissue Eng., Part C 2011, 18, 122-132.

(41) Hashizume, R.; Hong, Y.; Takanari, K.; Fujimoto, K. L.; Tobita, K.; Wagner, W. R. The Effect of Polymer Degradation Time on Functional Outcomes of Temporary Elastic Patch Support in Ischemic Cardiomyopathy. Biomaterials 2013, 34, 7353-7363.

(42) Hasan, A.; Memic, A.; Annabi, N.; Hossain, M.; Paul, A.; Dokmeci, M. R.; Dehghani, F.; Khademhosseini, A. Electrospun Scaffolds for Tissue Engineering of Vascular Grafts. Acta Biomater. 2014, 10, 11-25.

(43) de Valence, S.; Tille, J. C.; Mugnai, D.; Mrowczynski, W.; Gurny, R.; Möller, M.; Walpoth, B. H. Long Term Performance of Polycaprolactone Vascular Grafts in a Rat Abdominal Aorta Replacement Model. Biomaterials 2012, 33, 38-47.

(44) Lim, G. B. Interventional Cardiology: DREAMS of a Bioabsorbable Stent Coming True. Nat. Rev. Cardiol. 2013, 10, 120.
(45) Fan, R.; Bayoumi, A. S.; Chen, P.; Hobson, C. M.; Wagner, W. R.; Mayer, J. E., Jr; Sacks, M. S. Optimal Elastomeric Scaffold Leaflet Shape for Pulmonary Heart Valve Leaflet Replacement. J. Biomech. 2013, 46, 662-669.

(46) Driessen-Mol, A.; Emmert, M. Y.; Dijkman, P. E.; Frese, L.; Sanders, B.; Weber, B.; Cesarovic, N.; Sidler, M.; Leenders, J.; Jenni, R.; Grünenfelder, J.; Falk, V.; Baaijens, F. P.; Hoerstrup, S. P. Transcatheter Implantation of Homologous "Off-the-Shelf" Tissueengineered Heart Valves with Self-repair Capacity: Long-term Functionality and Rapid In Vivo Remodeling in Sheep. J. Am. Coll. Cardiol. 2014, 63, 1320-1329.

(47) De Kesela, C.; Lefevrea, C.; Nagy, J. B.; David, C. Blends of Polycaprolactone with Polyvinylalcohol: a DSC, Optical Microscopy and Solid State NMR Study. Polymer 1999, 40, 1969-1978.

(48) Sekosan, G.; Vasanthan, N. Morphological Changes of Annealed Poly- $\varepsilon$-caprolactone by Enzymatic Degradation with Lipase. J. Polym.Sci., B: Polym. Phys. 2010, 48, 202-211. 